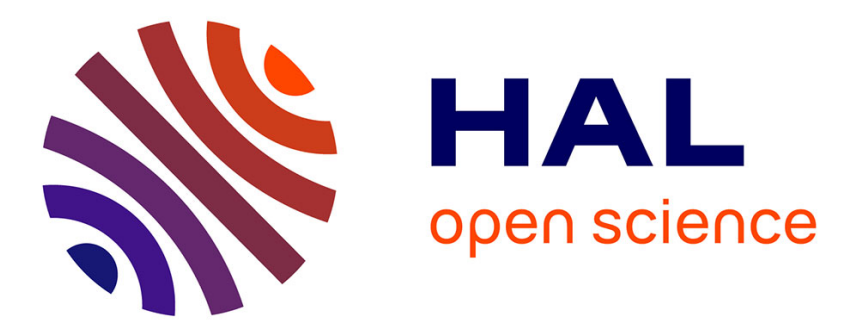

\title{
Genetic diversity of genotype 6 HCV infections in France epidemiology and consequences for treatment strategy
}

\author{
C. Pronier, F. Helene, D. Celine, P. Stanislas, F. Carrat, Vincent Thibault
}

\section{To cite this version:}

C. Pronier, F. Helene, D. Celine, P. Stanislas, F. Carrat, et al.. Genetic diversity of genotype 6 HCV infections in France epidemiology and consequences for treatment strategy. Journal of Hepatology, 2018, 68, pp.S291-S291. 10.1016/S0168-8278(18)30800-6 . hal-02118521

HAL Id: hal-02118521

https://hal-univ-rennes1.archives-ouvertes.fr/hal-02118521

Submitted on 14 Oct 2019

HAL is a multi-disciplinary open access archive for the deposit and dissemination of scientific research documents, whether they are published or not. The documents may come from teaching and research institutions in France or abroad, or from public or private research centers.
L'archive ouverte pluridisciplinaire HAL, est destinée au dépôt et à la diffusion de documents scientifiques de niveau recherche, publiés ou non, émanant des établissements d'enseignement et de recherche français ou étrangers, des laboratoires publics ou privés. 
PROF. VINCENT THIBAULT (Orcid ID : 0000-0001-6517-2901)

Article type : Original Paper

Genetic diversity of genotype 6 HCV infections in France: epidemiology and consequences for treatment strategy

Running Title: Genotype 6 HCV diversity and treatment

Charlotte Pronier ${ }^{1}$, Hélène Fontaine ${ }^{2}$, Céline Dorival ${ }^{3}$, Fabrice Carrat $^{3}$, Stanislas Pol $^{2}$, Vincent Thibault ${ }^{1}$ on behalf of the AFEF/ANRS Hepather study group

${ }^{1}$ Virology Univ Rennes, CHU Rennes, Inserm, EHESP, Irset (Institut de recherche en santé, environnement et travail) - UMR_S 1085, F-35000 Rennes, France

${ }^{2}$ Paris Descartes Univ., Hepatology, Hôpital Cochin, AP-HP, Inserm U1223 Institut Pasteur

${ }^{3}$ UMR S 1136-IPLESP, Sorbonne Univ., INSERM, Paris

Corresponding author:

- Vincent Thibault: vincent.thibault@chu-rennes.fr

- Pr Vincent THIBAULT, Virology, CHU Pontchaillou, 2 rue Henri Le Guilloux, 35000 Rennes, FRANCE

- tel: +33 299289059

- fax: +33299284159 


\section{Acknowledgments}

Authors are grateful to all patients who accepted to participate to the French ANRS-CO22-Hepather cohort.

\section{Abstract}

Genotype-6 hepatitis C virus (GT6-HCV) exhibits a high genetic diversity. GT6 prevalence, diversity and real-life response to treatment were studied among 14,603 HCV mono-infected patients from the French ANRS-CO22-Hepather cohort. NS3, NS5A and NS5B-HCV genes were amplified and sequenced for all GT6-infections identified in the database. Following phylogenic characterization, resistance associated substitution polymorphisms were identified. GT6-infected patients ( $n=36$; $0.25 \%)$ did not differ from patients infected with other genotypes with regard to gender, age or liver fibrosis. GT6e was the most prevalent (27.8\%), followed by 6a (22.2\%), 6q (11.1\%) and 6o (8.3\%). Each subtype $p$ and $x c$ were found in 2 patients (5.6\%) and subtypes $f / h / r$ and $t$ were each detected in one patient. Four strains (11.1\%) clustered with unclassified reference sequences. Concordant genotype determination throughout NS3, NS5A and NS5B-genes is consistent with lack of recombination within this genomic region. All but 3 patients were born in Asia, Cambodia (44.4\%), Vietnam (38.9\%) or Laos (8.3\%). GT6a was found in $42.8 \%$ of Vietnamese and $6 e$ in $37.5 \%$ of Cambodian. GT6q, $6 p$ and $6 r$ were only found in Cambodian patients. Resistance associated polymorphisms for each DAA classes were identified in baseline sequences. Twenty-seven patients were treated with sofosbuvir-based combinations and 3 with glecaprevir/pibrentasvir. All treated patients, whether naïve or previously treated, achieved a sustained viral response. In conclusion, GT6-infections are uncommon in France and their genetic diversity likely reflects infection within the country of origin. Despite residue variability at DAA resistance-associated positions, sustained viral response was obtained in all treated patients. 
Keywords: Direct acting antivirals - Subtypes - Resistance - Sustained viral response - Transmission

\section{Introduction}

Hepatitis C virus (HCV) is one of the major causative agents of chronic liver disease. Globally, in 2015, an estimated 71 million people were living with chronic HCV infection. The epidemic caused by HCV

affects all regions, with major distribution differences between and within countries ${ }^{1}$. HCV is classified into at least seven major genotypes (GT1-7) further subdivided into at least 86 subtypes ${ }^{2}$. A recent publication provides evidence for an additional eighth genotype ${ }^{3}$. HCV genotype 6 (GT6) is almost exclusively restricted to Southeast Asia (China, Vietnam, Cambodia and Laos) and it is occasionally found in migrant patients from endemic countries. Among the 7 HCV genotypes, GT6 exhibits the highest genetic diversity as assessed by intra-genotype pairwise distance and has been subdivided in at least 23 subtypes (a to $w)^{4,5}$.

Only two studies based on different HCV infected patient populations evaluated the specific liver disease natural history induced by GT6 with divergent results ${ }^{6,7}$. Seto et al., in a monocentric study from Hong-Kong, did not report any difference in the progression of the disease when comparing two groups of patients infected with either GT1 or GT6. By contrast, Lee et al. looking at Asian patients from the USA and Hong-Kong reported an increased risk of liver complications only in cirrhotic patients when compared to a GT1 infected control group. In summary, although based on few reports, GT6 infection natural history does not seem different to what observed with GT1. Whatever the evolution of the disease, the current WHO strategy is however to eliminate HCV infection from the planet by 2030 and availability of highly potent direct acting antivirals (DAA) justify to treat all screened positive patients independently of the liver lesions ${ }^{1}$. Until recent availability of pan-genotypic antiviral drugs in developed countries, HCV genotype has been a key marker to guide the best treatment option ${ }^{8}$. Considering the high genetic heterogeneity within GT6, it should be pointed out that limited data are available on both in vitro and in vivo antiviral activity of DAAs on 
this genotype. Data are even scarcer when considering subtypes. Indeed, most data on DAA efficacy to date have been derived from clinical trials conducted in Western countries, where GT6-HCV is rare and only few data sets exist for countries such as Cambodia and Vietnam with higher GT6 prevalence. Few in vitro studies have clearly identified significant difference in DAA potency according to GT6 subtypes or even selection of resistance ${ }^{9-12}$. Importantly, while their data validate DAA activity on subtype 6a prototypes, efficacy on less frequent subtypes is missing despite great polymorphism on residues known to influence DAA efficacy ${ }^{13}$. Thus, precise genetic characterization of HCV strains is useful to identify rare subtypes, likely those not included in large clinical trials, to detect clusters of transmission in high-risk groups or to identify polymorphisms associated with antiviral resistance.

GT6 infection rarely occurs in France and little is known about the mode of transmission of this genotype and its diversity in Western Europe. The prospective French ANRS CO22 Hepather cohort is a unique observational national multicenter setting to document, without bias, the distribution of GT6 infection in France and to study treatment response of GT6 infected patients. Through phylogenic analysis of NS3, NS5A and NS5B coding sequences, HCV-GT6 diversity was studied in patients included in the Hepather cohort and treatment efficacy was documented within each subtype.

\section{Materials and methods}

\section{Study design and participants}

The ANRS CO22 Hepather cohort ("Therapeutic options for hepatitis B and C: a French cohort") is a national multicenter prospective observational cohort study of patients with viral hepatitis B or C (this study is registered with ClinicalTrials.gov, number NCT01953458) (Refer to supplementary materials). We selected all patients with HCV genotype 6 infection who initiated treatment before December $2017(n=38)$. 


\section{HCV sequence amplification, sequencing and analysis}

For all GT6 HCV infections identified in the database, HCV subtype was determined on a plasma sample drawn at inclusion. NS3, NS5A and NS5B regions were RT-PCR amplified and sequenced using previously described protocols ${ }^{14}$. Each protein encoding sequences were aligned to HCV GT1-7 reference set downloaded from the ICTV website containing complete genomes of all subtypes described by Smith et al. and recently updated according to ICTV ${ }^{2}$. Based on each gene coding sequence alignments, phylogenic analyses were performed using MEGA 7.0 Software ${ }^{15}$. Briefly, the evolutionary history was inferred using the Neighbor-Joining method, the evolutionary distances were computed using the Maximum Composite Likelihood method and tree robustness was assessed through 500 replicate bootstrap tests.

Protein residues known to impact drug sensitivity were reported (refer to supplementary materials).

\section{Results}

\section{Patient characteristics}

Data mining on Hepather database initially identified 38 cohort participants with GT6 infection. For 2 patients, HCV sequence determination revealed a misclassification and allowed for reclassification as genotype 1a and 3 (Fig. 1). Overall, 36 patients out of 14,603 (0.25\%) were confirmed as infected with a GT6 virus (Table 1). Patients had a mean age of 59 years, $44.4 \%$ were male, most (33/36; 91.7\%) were born in Southeast Asia (Cambodia, Vietnam, Laos) and the 3 remaining were European. While transfusion could explain $16.7 \%$ of HCV transmission and injecting drug use, tattooing and piercing accounted each for $5.6 \%$ of the cases. The remaining routes of transmission were unknown, even though most patients reported longer periods than tourism travels in their country of birth. Noteworthy, 23 patients (64\%) had an HBV serological profile indicating past HBV infection (anti-HBC positive). Cirrhotic patients represented 13 (36\%) of the GT6 infected subjects and mild fibrosis (Metavir score $\leq 2$ ) was documented for 16 patients $(44 \%)$. Compared to the whole cohort, 
genotype 6 infected patients came more likely from Asia $(p<0.0001)$, were more often treatmentnaïve $(p=0.04)$ and had a higher viral load $(p=0.0004)$.

\section{HCV GT6 subtype distribution}

Sequencing was successful for all NS3 and NS5A encoding regions while failure occurred for 7 (19\%) NS5B genes (figure 1). Failure was attributed to non-optimal RT-PCR conditions and eventually lack of material to repeat the experiment. Phylogenic analysis allowed subtype determination for 32 (89\%) strains while 4 sequences did not cluster with enough confidence into confirmed subtypes. Whatever analyzed gene, phylogenic analyses provided concordant classification into 10 different subtypes. A representative NS5A phylogenic tree is shown on figure 2. Among sequences that did not cluster with assigned subtypes, F4 presented similarities with JX183550 and C2 was close to KJ567649 and KJ567650. Strains A4 and H4 were more distant to any reference sequences, although some proximity was noticed with subtype 60 and $6 q$, respectively. The most prevalent subtypes were $6 e$ $(10 ; 27.8 \%), 6 a(8 ; 22.2 \%)$, and $6 q(4 ; 11.1 \%)$. Interestingly, among notified subtypes $(n=20)$ as registered within the database, the initially determined subtype was only confirmed through sequencing for 9 (45\%) samples.

Despite a large genotype diversity within each country, GT6q $(n=4)$ was only found in patients from Cambodia where subtype 6 e $(n=6 ; 37.5 \%)$ is also predominant. In patients from Vietnam, GT6a $(n=6$; 42.8\%) was the most prevalent (Figure 3).

\section{Polymorphism}

GT6 polymorphisms were studied for critical residues (Resistance Associated Substitutions or RAS) known to confer resistance to currently available DAA (figure 4). For each viral target (NS3 and NS5A), a protein alignment of 76 reference sequences covering all GT6 subtypes was generated and key residues were compared to those most prevalent in genotype 1a strains. For the NS3 protein, positions $41,43,54,55,155,156$ were similar to the one found in GT1a and mostly conserved whatever the subtype (Fig. 4a). Positions 56 and 168 were also frequently conserved with only few 
sequences with a unique change either Y56F or D168E. More diversity was noted on residues 36,80 , 122 and 170, the highest number of different residues occurred on positions 36 and 80 . While the most prevalent residues at RAS positions were identical to those of GT1a, position 122 was the less conserved (S122T/N).

For protein NS5A, residues 32 and 38 were identical to GT1a strain and residue 92 had the lowest variability (fig. 4b). By contrast, positions $28,30,58$ and to a lower extent 24,31 and 93 showed very frequent polymorphisms with up to 8 different variations for a unique position. The most prevalent changes were concomitantly found at residues M28V, H58P and Y93T. Noteworthy, S282T NS5B residue reported to confer decreased sensitivity to sofosbuvir was never found throughout analyzed Gt6-sequences and residues L320 and V321, possibly impacting sofosbuvir activity were also conserved throughout all sequences. Residue L159, located upstream, was not covered by our sequencing approach.

\section{Real life treatment efficacy}

As the cohort was initiated in 2012, several lines of treatment options have been recommended over the course of time. In our cohort, one-third (13/36) of the patients had received a previous treatment, either IFN-based for the majority $(92.3 \%$; $n=12)$ or DAA-based for one patient (GZR/EBR) (Table 2). Among the patients who had received IFN, most $(n=8 ; 66.7 \%)$ were relapsers and one was non-responder. Treatment was initiated for 30 patients after inclusion in the Hepather cohort, 18 were naïve and 12 were previously treated. Most patients $(n=27 ; 90 \%)$ received a SOF-containing DAA combination treatment, either associated with an anti-NS5A $(n=20 ; 66.7 \%)$, an anti-NS3 $(n=1$; $3.3 \%$ ) or both ( $n=2 ; 6.7 \%)$. Sofosbuvir combined with IFN plus ribavirin was given to 4 patients and the remaining 3 patients received a Glecaprevir/Pibrentasvir combination. Median duration of DAA treatment was 12 weeks. 
All treated patients achieved SVR12. Among NS5A inhibitors, ledipasvir $(n=11)$ was the most used, followed by daclatasvir $(n=7)$, velpatasvir $(n=4)$ and pibrentasvir $(n=3)$. All cirrhotic patients, 7 treatment-experienced and 6 treatment naïve, were successfully treated. Noteworthy, no serious adverse event or treatment discontinuation was reported for treated patients even in patients with advanced liver fibrosis.

\section{Discussion}

Although awareness on hepatitis $\mathrm{C}$ infection as a preventable infectious disease is significantly decreasing its incidence worldwide, people who inject drugs (PWID) and migration fluxes from highly endemic countries remain major sources of new infections ${ }^{16}$. As genotype 6 infected patients are rarely encountered in France, a systematic study based on the largest observational cohort in France (ANRS CO22 Hepather) was conducted to describe the characteristics of HCV-GT6 infected patients and the efficacy of new treatments on this specific population.

While recent DAA based treatment options appear fully active on circulating GT6 strains, a large diversity of GT6 viruses was characterized. This heterogeneity likely reflects imported cases from disparate sources, rather than infections acquired in France through one specific mode of transmission. The absence of clear transmission cluster within GT6 subtypes tends to support the hypothesis that most transmissions occurred in the country of origin rather than locally through common risk factors. Distribution of many subtypes in the Hepather cohort directly reflects the subtype distribution in the patient's country of origin ${ }^{5}$. Indeed, all cases but $3(8 \%)$ were born in GT6 endemic areas. Among the 3 patients potentially infected in France, one was through injection drug use, a second by possible nosocomial transmission and for the last one, no specific risk was found. The identified strains for these 3 patients belonged to 2 different subtypes ( 0 and a); the two 60 strains being not phylogenetically related. Noteworthy, beside geographic origins, Hepather GT6 
infected patients do not differ from those infected with other genotypes in terms of age, gender and liver disease.

The number of GT6 infected patients identified in the Hepather cohort is consistent with different prevalence studies performed in France, reporting around $0.2 \%$ of patients being infected by this genotype. As previously described, genotype characterization may sometimes be impaired by the methodological approach. Indeed, 2 out of the 38 (5.2\%) patients were erroneously documented as genotype 6 infected, likely reflecting either a wrong input or a bias due to the genotyping technical approach, especially if targeting the 5 '-non coding region ${ }^{17-19}$. Thus, the true prevalence of GT6 could potentially be slightly underestimated when the strains are misclassified as GT1 when using 5'-non coding region analysis. Using sequencing approaches based on more informative genomic regions should be promoted, particularly to document transmission in at-risk populations ${ }^{20}$. For all sequenced strains, concordant phylogenic analyses whatever the chosen region, NS3, NS5A or NS5B were obtained and most sequences clustered with GenBank available data. Routine sequencing analysis is also useful to document hypothetical new subtypes or baseline RAS that may impair treatment efficacy, particularly in difficult to treat patients. Four strains could indeed not be classified according to the most recent ICTV nomenclature and showed an important genetic distance from available classified sequences ${ }^{2}$. These strains presented no match either with the recently described GT6xg subtype ${ }^{21}$. Interestingly, most recent studies that reported gt6 subtype distribution from large clinical trial on new DAA did not report any "o" or "f" subtypes and no firm conclusion can be drawn from these trials on DAA efficacy on these less prevalent subtypes ${ }^{22,23}$. Indeed, McPhee et al. report in vitro high level of resistance to velpatasvir in replicons carrying both NS5A L28T/R30S RAS, a common motif found exclusively in subtype $6 \mathrm{f}$ and also found in one of our patients ${ }^{11}$. We believe awareness should be promoted when treating uncommon subtypes that may not be as susceptible to the so-called "pan-genotypic" current combinations, even though current real-world efficacy data are very reassuring. 
Data on antiviral efficacy on GT6 natural strains are very scarce. Yet, the large heterogeneity of GT6 strains even at the DAA target protein level raises the possibility of reduced antiviral susceptibility compared to what observed on GT1 viruses. Indeed, an important polymorphism could be documented on selected reference sequences belonging to all GT6 subtypes at classical RAS positions (Figure 4). While results from large clinical trials that included few GT6 patients usually report good efficacy on this genotype, few studies, using mostly artificial chimeric constructs based on a GT6a backbone reported in vitro DAA activity. Regarding antiprotease activity, glecaprevir had similar antiviral activity compared to grazoprevir against GT 6 a and 6e tested replicons, with $\mathrm{EC}_{50}$ values of 0.86 and $0.21 \mathrm{nM}$, respectively; values similar to what observed for GT1a strains. However, selected glecaprevir RAS at position D168A/H/V reduced the susceptibility to glecaprevir by 38 to 146 -fold in cells containing a genotype 6a replicon. In the same system, grazoprevir demonstrated also a 247 to 1,048-fold reduced activity ${ }^{24,25}$. More recently, a publication looking at efficacy of a 12 week grazoprevir/elbasvir treatment, reported an SVR in only $62.9 \%$ (22/35) of a subgroup of GT6 patients, demonstrating the lack of power of this combination for this genotype ${ }^{26}$. Analysis of GT6 NS3 from different subtypes identified a rare D168E polymorphism not described as modifying anti-NS3 susceptibility and no such polymorphism was found on our patient sequences. Our findings and reports from the literature are thus quite reassuring in term of second generation anti-NS3 efficacy on all GT6 subtypes, with no evidence for polymorphism significantly modifying DAA potency ${ }^{11}$. Regarding anti-NS5A potency, daclatasvir was as effective in vitro against a GT6a hybrid replicon (EC 50 $74 \mathrm{pM})$ as on a similar GT1a construct ${ }^{27}$. Except for P32L leading to a high level of resistance $\left(\mathrm{EC}_{50}\right.$ $250 \mathrm{nM}$ ), the following RAS, Q24H, L31M, P32S and T58A/S are known to confer modest levels of resistance against daclatasvir $\left(\mathrm{EC}_{50}\right.$ 2-44 $\left.\mathrm{nM}\right)$. In our set, RAS L31M was identified in one case and T58A, contributing to lower treatment efficacy, in 5 patients. Interestingly, substitution T58A is found in all 6o subtypes from this study as in the included reference sequences and could therefore represent a specific protein signature for this subtype. However, it should be pointed out that patients presenting this T58A polymorphism and treated with a daclatasvir containing combination 
$(n=3)$ did clear the virus. Ledipasvir has a potent in vitro antiviral activity against GT-1a replicons with $\mathrm{EC}_{50}$ around $0.031 \mathrm{nM}$. However, on GT6, the study by Cheng et al. describes a comparatively lower activity of ledipasvir, with a decreasing efficacy for subtypes 6a $\left(\mathrm{EC}_{50}\right.$ of $\left.1.1 \mathrm{nM}\right)$ even more pronounced for GT-6e with an $\mathrm{EC}_{50}$ of $264 \mathrm{nM}^{9}$. Interestingly, $3 \mathrm{GT6e}$ cirrhotic patients included in the Hepather cohort and treated with sofosbuvir-ledipasvir, achieved SVR. McPhee et al. also describe a double variant NS5A L28A/R30S with a more than 10,000 fold reduced sensitivity to ledipasvir ${ }^{11}$. This motif was detected in one GT6f patient who had a sustained virological response to 3 months of SOF-LED + ribavirin combination treatment. Antiviral activity of pibrentasvir was unaffected against a panel of replicons integrating NS5A genes from infected patients with subtypes $6 a, 6 e$ or $6 \mathrm{p}(\mathrm{n}=5)$ with an $\mathrm{EC}_{50}$ around $0.7 \mathrm{pM}$, despite polymorphisms detected in some of these clinical samples ${ }^{12,28}$. By contrast, a slight difference in velpatasvir antiviral activity has been reported between $6 a$ and $6 e$ subtypes, with $\mathrm{EC}_{50}$ of 6 and $130 \mathrm{pM}$ respectively ${ }^{29}$. Moreover, a valine substitution at position L31 could also potentially affect velpatasvir activity on GT6a as recently demonstrated by Pham et al. ${ }^{10}$. Analyses performed on reference sequences and on sequences from our patients never identified such L31V polymorphism but the residue change L31M/I was found in 2 patients. Although exceptional, clinical sofosbuvir resistance involves the selection of a S282T RAS that leads to increased EC50 against the molecule but also major impairment of the virus replication fitness ${ }^{30}$. Whether on selected GT6 reference sequences or sequences generated from the Hepather patients, this RAS was never detected, suggesting that sofosbuvir constitutes a solid cornerstone for GT6 combination treatments. A recent single study, describing a S282T prevalence of $20.7 \%$ in a Chinese cohort infected with GT6a-HCV, should however raises our attention on the possible emergence of such variants ${ }^{31}$. Of note, no change was observed on residues $L 320$ and V321, involved by some authors with decreased sensitivity to sofosbuvir. L159 was however not covered by our sequencing approach. 
Several conclusions can be drawn from our experience. First, the overall real life treatment efficacy was $100 \%$ in these GT6 infected patients, a higher rate than what was found in 3 studies using a SOF/LED combination approach ${ }^{30-32}$. As this retrospective study is based on a prospective cohort, it was not possible to gather more details on viral kinetics or adverse events but the observed high SVR rate certainly validates the French successive updated recommendations to manage HCV infected patients throughout the inclusion period (2012-2017). Consequently, all patients did not receive the same combination strategy over time, which prevented us to better document treatment response according to well described SVR predictors. Second, the frequent polymorphism linked to subtype diversity had no effect on clinical treatment efficacy. This finding is quite surprising as several residue positions known to interfere with treatment efficacy in other genotypes were detected in a number of strains. As a note of caution, one should emphasize that all but one patient received at least 12 weeks of treatment. Only one patient received an 8-week combination of SOF-VEL-VOX. Twelveweek treatment duration is not anymore the standard of care and according to recent clinical trials, 8 weeks are probably enough to clear the virus in absence of cirrhosis for some combinations. Further studies are encouraged to document treatment efficacy in genotype 6 infected patients that will be eligible to 8 weeks treatment with pangenotypic combinations.

On a practical point of view, our study based on a large and diverse patient population conveys a very positive message regarding the efficacy of current treatment strategies to cure any GT6 subtype infection, at least with a 12-week regimen. Despite abundant polymorphisms on RAS, approved DAA combinations used according to current recommendations lead to very high rate of sustained viral response, $100 \%$ in our real-life cohort. With the recent availability of pangenotypic combinations, virus genotyping presents less clinical relevance in treatment naïve patients. Yet, documentation of RAS polymorphisms for rare genotypes could be relevant as little proof of evidence exists regarding efficacy of these new treatments on such strains. Moreover, sequence determination is useful to document cluster of transmissions, particularly in high-risk population. Our study demonstrates that 
most genotype 6 infections in France, also possibly in other European countries, does not result from transmissions at the country level but rather from imported cases.

\section{Funding}

This work was supported by INSERM-ANRS (France REcherche Nord\&sud Sida-vih Hepatites), ANR (Agence Nationale de la Recherche), DGS (Direction Générale de la Santé) and Gilead, Abbvie, MSD, Janssen, BMS, Roche.

\section{Conflict of interest disclosure statement}

H. Fontaine declares participation at therapeutic trials and personal fees from Roche, Janssen, Gilead, Bristol Myers Squibb, MSD and Abbvie

S. Pol has received consulting and lecturing fees from Bristol-Myers Squibb, Janssen, Gilead, MSD, Abbvie and grants from Bristol-Myers Squibb, Gilead, Roche and MSD.

V. Thibault has received travel grants and lecturing fees from Bristol-Myers Squibb, Janssen, Gilead, MSD and Abbvie.

C. Pronier, C. Dorival and F. Carrat declare no conflict of interest for the presented work. 


\section{References}

1. World Health Organization, Global Hepatitis Program. Global Hepatitis Report, 2017.; 2017. http://apps.who.int/iris/bitstream/10665/255016/1/9789241565455-eng.pdf?ua=1. Accessed April 25, 2017.

2. Smith DB, Bukh J, Kuiken C, et al. Expanded classification of hepatitis C virus into 7 genotypes and 67 subtypes: Updated criteria and genotype assignment web resource Hepatology. 2014;59(1):318-327. doi:10.1002/hep.26744

3. Borgia SM, Hedskog C, Parhy B, et al. Identification of a novel hepatitis C virus genotype from Punjab, India: expanding classification of hepatitis $C$ virus into 8 genotypes. The Journal of Infectious Diseases. June 2018. doi:10.1093/infdis/jiy401

4. Thong VD. Hepatitis C virus genotype 6: Virology, epidemiology, genetic variation and clinical implication. World Journal of Gastroenterology. 2014;20(11):2927. doi:10.3748/wjg.v20.i11.2927

5. Li C, Barnes E, Newton PN, et al. An expanded taxonomy of hepatitis C virus genotype 6: Characterization of 22 new full-length viral genomes. Virology. 2015;476:355-363. doi:10.1016/j.virol.2014.12.025

6. Seto $\mathrm{W}-\mathrm{K}$, Lai C-L, Fung J, et al. Natural history of chronic hepatitis C: genotype 1 versus genotype 6. Journal of Hepatology. 2010;53(3):444-448. doi:10.1016/j.jhep.2010.04.009

7. Lee $\mathrm{M}-\mathrm{H}, \mathrm{Hsiao} \mathrm{TI}$, Subramaniam SR, et al. HCV genotype 6 increased the risk for hepatocellular carcinoma among Asian patients with liver cirrhosis. The American Journal of Gastroenterology. 2017;112(7):1111-1119. doi:10.1038/ajg.2017.123

8. European Association for Study of Liver. EASL Clinical Practice Guidelines: management of hepatitis C virus infection. J Hepatol. 2014;60(2):392-420. doi:10.1016/j.jhep.2013.11.003

9. Cheng $\mathrm{G}$, Tian $\mathrm{Y}$, Doehle $\mathrm{B}$, et al. In vitro antiviral activity and resistance profile characterization of the hepatitis C virus NS5a inhibitor ledipasvir. Antimicrobial Agents and Chemotherapy. 2016;60(3):1847-1853. doi:10.1128/AAC.02524-15

10. Pham LV, Ramirez S, Gottwein JM, et al. HCV genotype 6a escape from and resistance to velpatasvir, pibrentasvir, and sofosbuvir in robust infectious cell culture models. Gastroenterology. 2018;154(8):2194-2208.e12. doi:10.1053/j.gastro.2018.02.017

11. McPhee F, Ueland J, Vellucci V, Bowden S, Sievert W, Zhou N. Impact of preexisting hepatitis c virus genotype 6 NS3, NS5A, and NS5B polymorphisms on the in vitro potency of direct-acting antiviral agents. Antimicrob Agents Chemother. 2019;63(4). doi:10.1128/AAC.02205-18

12. Schnell $G$, Krishnan $P$, Tripathi $R$, et al. Hepatitis $C$ virus genetic diversity by geographic region within genotype 1-6 subtypes among patients treated with glecaprevir and pibrentasvir. PLoS ONE. 2018;13(10):e0205186. doi:10.1371/journal.pone.0205186

13. Sorbo MC, Cento V, Di Maio VC, et al. Hepatitis C virus drug resistance associated substitutions and their clinical relevance: Update 2018. Drug Resistance Updates. 2018;37:17-39. doi:10.1016/j.drup.2018.01.004 
14. Andre-Garnier E, Besse B, Rodallec A, et al. An NS5A single optimized method to determine genotype, subtype and resistance profiles of Hepatitis C strains. Jhaveri R, ed. PLOS ONE. 2017;12(7):e0179562. doi:10.1371/journal.pone.0179562

15. Kumar S, Stecher G, Tamura K. MEGA7: Molecular Evolutionary Genetics Analysis version 7.0 for bigger datasets. Molecular Biology and Evolution. 2016;33(7):1870-1874. doi:10.1093/molbev/msw054

16. Bruggmann $\mathrm{P}$, Berg $\mathrm{T}, \varnothing$ vrehus ALH, et al. Historical epidemiology of hepatitis $\mathrm{C}$ virus (HCV) in selected countries. J Viral Hepat. 2014;21 Suppl 1:5-33. doi:10.1111/jvh.12247

17. Yang R, Cong X, Du S, Fei R, Rao H, Wei L. Performance comparison of the Versant HCV genotype 2.0 assay (LiPA) and the Abbott Realtime HCV genotype II assay for detecting hepatitis C virus genotype 6. J Clin Microbiol. 2014;52(10):3685-3692. doi:10.1128/JCM.0088214

18. Larrat S, Poveda J-D, Coudret C, et al. Sequencing assays for failed genotyping with the versant hepatitis C virus genotype assay (LiPA), version 2.0. J Clin Microbiol. 2013;51(9):2815-2821. doi:10.1128/JCM.00586-13

19. Némoz B, Roger L, Leroy V, Poveda J-D, Morand P, Larrat S. Evaluation of the $\operatorname{cobas}^{\circledR}$ GT hepatitis $C$ virus genotyping assay in G1-6 viruses including low viral loads and LiPA failures. Yu M-L, ed. PLOS ONE. 2018;13(3):e0194396. doi:10.1371/journal.pone.0194396

20. Chueca N, Rivadulla I, Lovatti R, et al. Using NS5B sequencing for hepatitis C virus genotyping reveals discordances with commercial platforms. PLOS ONE. 2016;11(4):e0153754. doi:10.1371/journal.pone.0153754

21. Ye $M$, Chen $X$, Wang $Y$, Duo L, Zhang $C$, Zheng $Y$-T. Identification of a new HCV subtype $6 x g$ among injection drug users in Kachin, Myanmar. Frontiers in Microbiology. 2019;10. doi:10.3389/fmicb.2019.00814

22. Boyd SD, Harrington P, Komatsu TE, et al. HCV genotype 4, 5 and 6: Distribution of viral subtypes and sustained virologic response rates in clinical trials of approved direct-acting antiviral regimens. J Viral Hepat. 2018;25(8):969-975. doi:10.1111/jvh.12896

23. Hezode C, Reau N, Svarovskaia ES, et al. Resistance analysis in patients with genotype 1-6 HCV infection treated with sofosbuvir/velpatasvir in the phase III studies. J Hepatol. 2018;68(5):895903. doi:10.1016/j.jhep.2017.11.032

24. Pham LV, Jensen SB, Fahnøe U, et al. HCV genotype 1-6 NS3 residue 80 substitutions impact protease inhibitor activity and promote viral escape. J Hepatol. 2019;70(3):388-397. doi:10.1016/j.jhep.2018.10.031

25. Jensen SB, Serre SBN, Humes DG, et al. Substitutions at NS3 Residue 155, 156, or 168 of Hepatitis $C$ Virus Genotypes 2 to 6 Induce Complex Patterns of Protease Inhibitor Resistance. Antimicrobial Agents and Chemotherapy. 2015;59(12):7426-7436. doi:10.1128/AAC.01953-15

26. George J, Burnevich E, Sheen I-S, et al. Elbasvir/grazoprevir in Asia-Pacific/Russian participants with chronic hepatitis C virus genotype 1, 4, or 6 infection. Hepatol Commun. 2018;2(5):595606. doi:10.1002/hep4.1177 
27. Wang C, Jia L, O'Boyle DR, et al. Comparison of daclatasvir resistance barriers on NS5A from hepatitis $C$ virus genotypes 1 to 6: implications for cross-genotype activity. Antimicrobial Agents and Chemotherapy. 2014;58(9):5155-5163. doi:10.1128/AAC.02788-14

28. Ng TI, Krishnan P, Pilot-Matias T, et al. In vitro antiviral activity and resistance profile of the next-generation hepatitis C virus NS5a inhibitor pibrentasvir. Antimicrobial Agents and Chemotherapy. 2017;61(5):e02558-16. doi:10.1128/AAC.02558-16

29. Cheng G, Yu M, Peng B, et al. GS-5816, a second generation HCV NS5a inhibitor with potent antiviral activity, broad genotypic coverage and a high resistance barrier. Journal of Hepatology. 2013;58:S484-S485. doi:10.1016/S0168-8278(13)61192-7

30. Xu S, Doehle B, Rajyaguru S, et al. In vitro selection of resistance to sofosbuvir in HCV replicons of genotype 1 to 6 . Antiviral Therapy. 2017;22(7):587-597. doi:10.3851/IMP3149

31. Li Z, Liu Y, Zhang $Y$, et al. Naturally occurring resistance-associated variants to hepatitis $C$ virus direct-acting antiviral agents in treatment-naive HCV genotype 6a-infected patients. BioMed Research International. 2017;2017:1-8. doi:10.1155/2017/9849823

32. Gane EJ, Hyland RH, An D, et al. Efficacy of ledipasvir and sofosbuvir, with or without ribavirin, for 12 weeks in patients with HCV genotype 3 or 6 infection. Gastroenterology.

2015;149(6):1454-1461.e1. doi:10.1053/j.gastro.2015.07.063 
Table 1. Baseline characteristics of patients

\begin{tabular}{|c|c|c|c|}
\hline & n (\%) & (cohort-n=11,863) & P-value \\
\hline Age $(y r)$ Mean \pm SD & $59 \pm 12$ & $57 \pm 11$ & 0.17 \\
\hline Gender male & $16(44)$ & $6638(56)$ & 0.16 \\
\hline \multicolumn{4}{|l|}{ Country of birth } \\
\hline Cambodia & $16(44.4)$ & $39(0.3)$ & \multirow[t]{4}{*}{$<0.000$} \\
\hline Vietnam & $14(38.9)$ & $64(0.5)$ & \\
\hline France & $3(8.3)$ & $8348(70.8)$ & \\
\hline Laos & $3(8.3)$ & $12(0.1)$ & \\
\hline \multicolumn{4}{|l|}{ Route of transmission } \\
\hline Known risk factor & $22(61.1)$ & $8522(71.8)$ & \multirow[t]{2}{*}{0.16} \\
\hline Unknown risk factor & $14(38.9)$ & $3341(28.2)$ & \\
\hline \multicolumn{4}{|l|}{ Fibrosis score } \\
\hline$\leq \mathrm{F} 2$ & $16(44.4)$ & $5188(43.7)$ & \multirow[t]{4}{*}{0.57} \\
\hline F3 & $6(16.7)$ & $1419(12.0)$ & \\
\hline F4 & $13(36.1)$ & $4266(36.0)$ & \\
\hline Unknown & $1(2.8)$ & $990(8.4)$ & \\
\hline \multicolumn{4}{|l|}{ Treatment history } \\
\hline naïve patients & $23(63.9)$ & $5417(45.7)+$ & \multirow[t]{2}{*}{0.04} \\
\hline experienced patients & $13(36.1)$ & $6395(53.9)$ & \\
\hline Interferon containing regimen & $12(92.3)$ & $4829(96.8) \ddagger$ & 0.35 \\
\hline GZR/EBR & $1(7.7)$ & IFN-free:160 (3.2) & \\
\hline Median RNA HCV inclusion (log IU/ml), [IQR] & $6.42[0.81]$ & $6.03 \S[0.94]$ & 0.0004 \\
\hline
\end{tabular}


Table 2: subtype distribution and treatment regimen

\section{Determined subtype}

- $6 a$

$8(22.2)$

- $6 \mathrm{e}$

$10(27.8)$

- $6 f$

$1(2.8)$

- $6 h$

$1(2.8)$

- 60

- $6 p$

- $6 \mathrm{q}$

- $6 r$

- $6 \mathrm{t}$

$1(2.8)$

- $6 x c$

$1(2.8)$

- 6 ?

Treatment $(n=30)$

- Interferon + ribavirin + Sofosbuvir

- Sofosbuvir + anti-NS5A

$$
\text { (VEL } n=3 ; \text { DCV } n=5 ; \text { LDV } n=10 \text { ) }
$$

- Sofosbuvir + anti-NS5A + ribavirin

- Sofosbuvir + anti-NS5A + anti-NS3

- Sofosbuvir + anti-NS5A + anti-NS3 + ribavirin

- Sofosbuvir + anti-NS3

- Glecaprevir + Pibrentasvir

Median treatment duration

$$
\text { (weeks), [Min-Max] }
$$


Legend to figures

Figure 1: General study outline

Through database mining, 38 patients were identified as HCV-GT6 infected and inclusion samples were collected for genotype confirmation through HCV genome sequencing.

Figure 2: Genotype 6 NS5A coding sequence phylogenic tree

GT6 Reference sequences (open circles) obtained from GenBank and 36 GT6 Hepather sequences (plain triangles) were aligned and the evolutionary distances were computed using the maximum composite likelihood method. The evolutionary history was inferred using the Neighbor-Joining method and a bootstrap value of 500 . The tree is drawn to scale indicated at the bottom left. Genotype 6 subtypes corresponding to Hepather sequences are circled and called when possible.

Figure 3: genotype 6 subtype distribution according to country of birth. Each subtype is represented with different motives or shade of grey and total numbers are indicated after the subtype.

Figure 4: NS3(a) and NS5A(b) protein polymorphisms for common RAS are represented as Logo charts. Residues corresponding to GT 1a reference sequence at indicated position are represented underneath. Analysis was performed on 76 ICTV reference sequences covering all different subtypes and including some unclassified strains. 


\section{French HEPATHER Cohort} $n=14,603$ patients

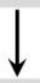

Gt 6 identified patients

$$
n=38
$$

Gt 6 confirmed patients $\mathrm{n}=2($ Gt $1 \mathrm{a} \& 3)$

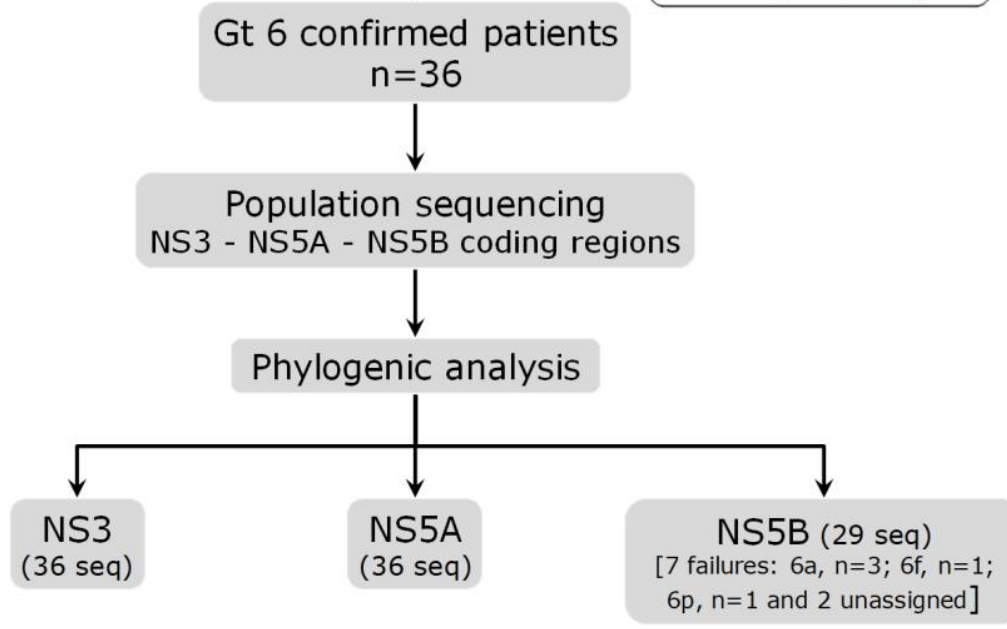




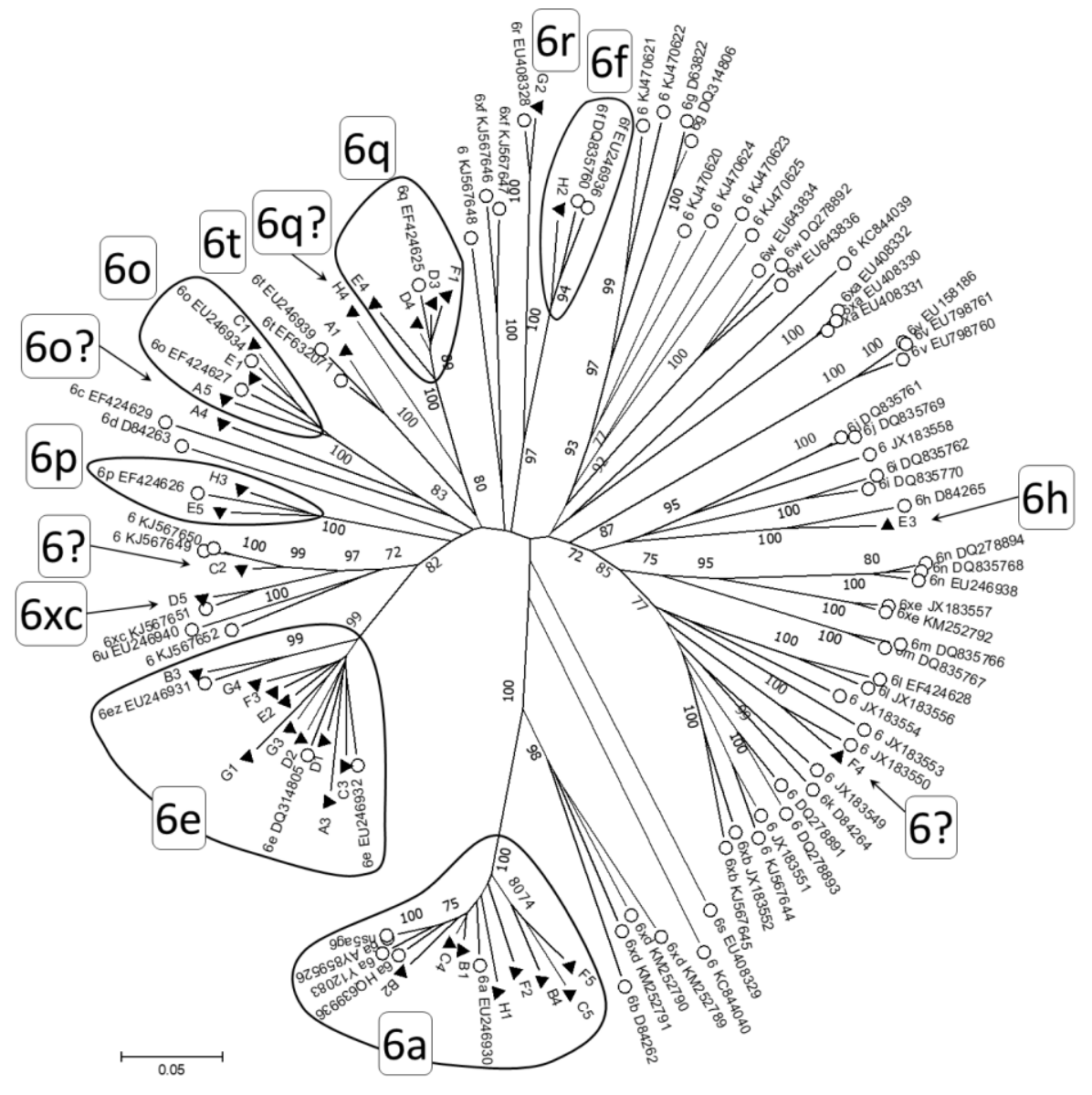




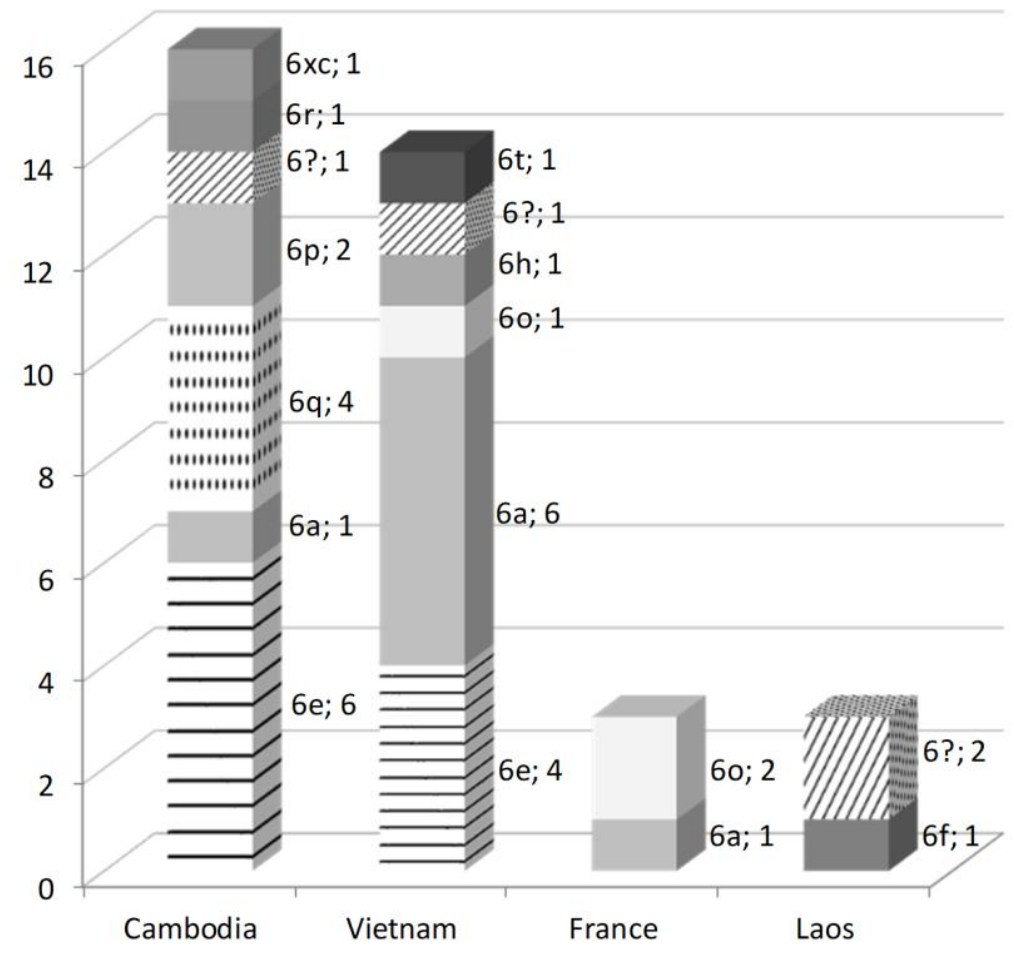




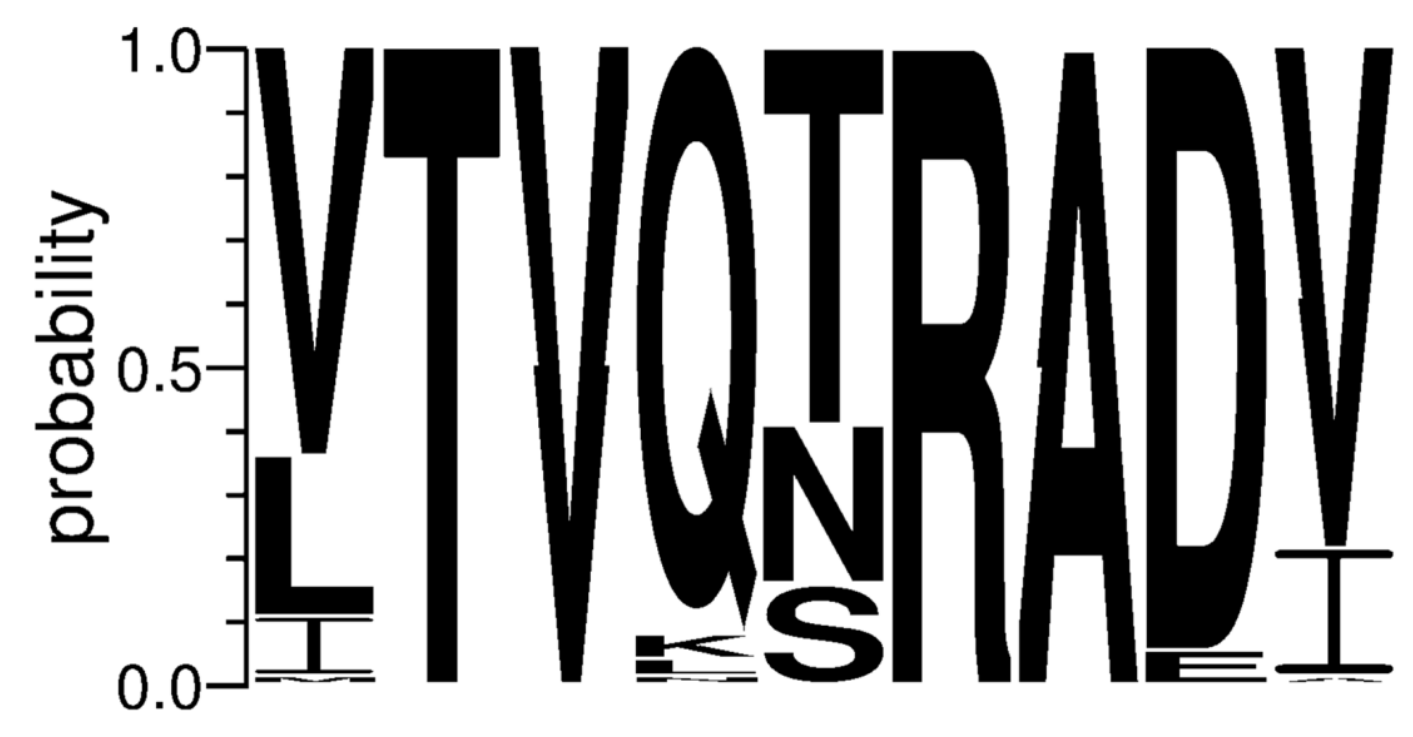

$\begin{array}{cccccccccc} & 36 & 54 & 56 & 80 & 122 & 155 & 156 & 168 & 170 \\ \text { Gt1a residues } & V & T & Y & Q & S & R & A & D & V\end{array}$ 


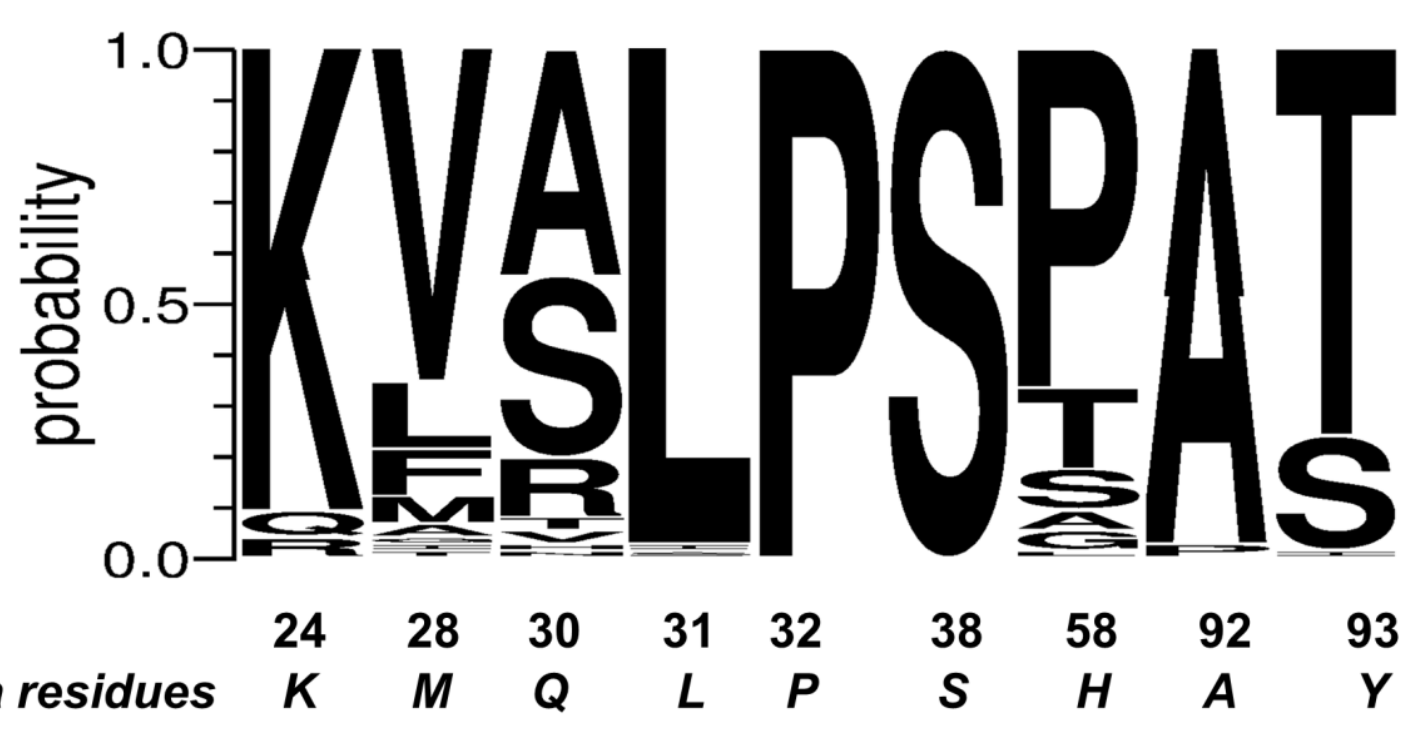

